\title{
What Happened to the Flu and RSV?
}

\section{Susan Hepworth}

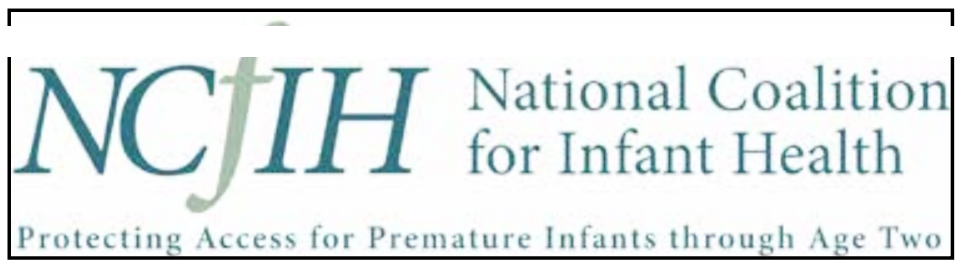

The National Coalition for Infant Health is a collaborative of more than 200 professional, clinical, community health, and family support organizations focused on improving the lives of premature infants through age two and their families. NCflH's mission is to promote lifelong clinical, health, education, and supportive services needed by premature infants and their families. NCfIH prioritizes safety of this vulnerable population and access to approved therapies.
Wash your hands. Cover your nose. It's familiar advice, but more people seem to be following it in the wake of a global pandemic. In fact, the behaviors aimed at helping to slow the spread of COVID-19 have also curbed the spread of other common viruses.

"Wash your hands. Cover your nose. It's familiar advice, but more people seem to be following it in the wake of a global pandemic. In fact, the behaviors aimed at helping to slow the spread of COVID-19 have also curbed the spread of other common viruses."

Cases of flu, for example, remain unusually low this year. During

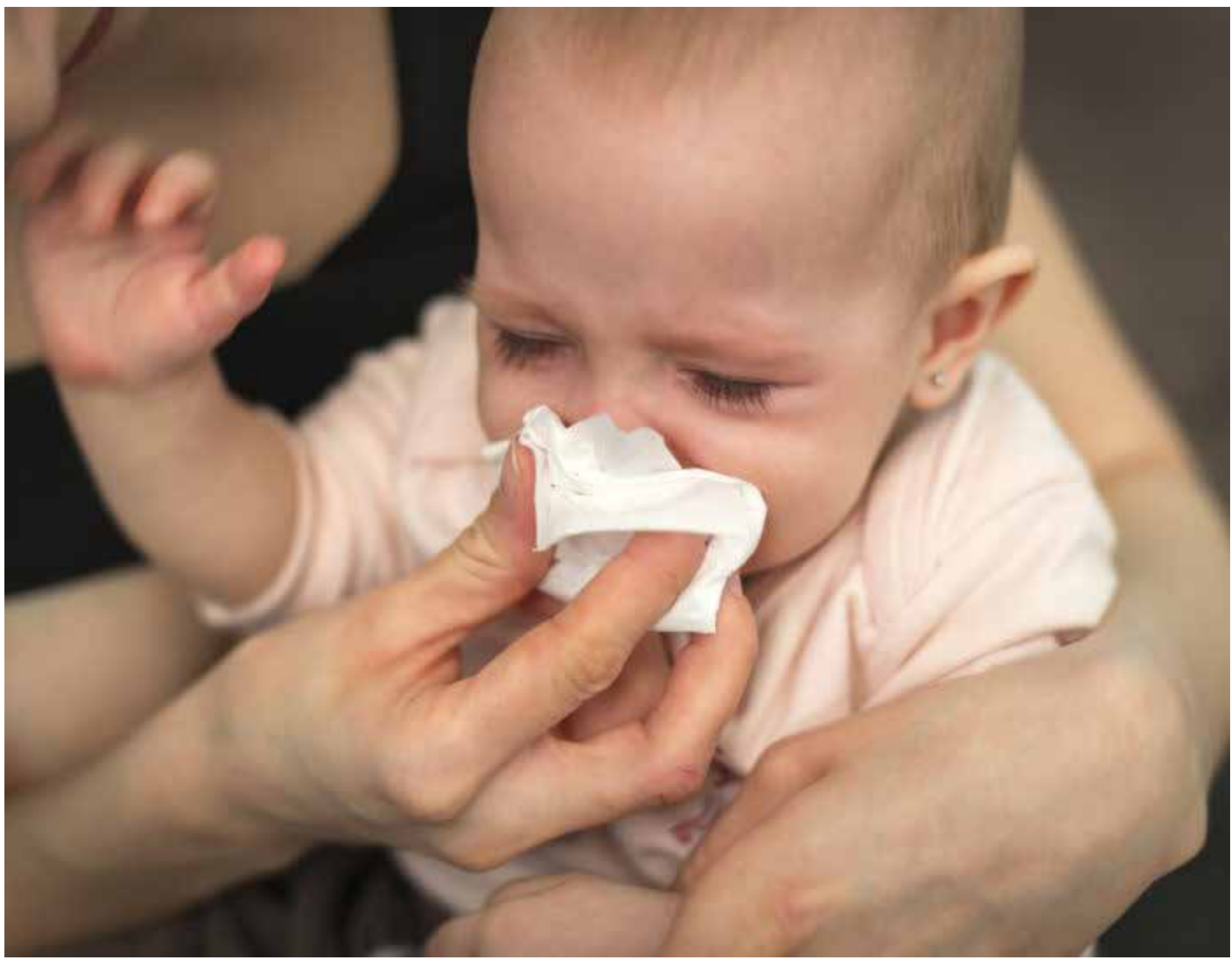


the 2019 flu season, the CDC reported more than 65,000 cases of influenza nationwide. During the same period this flu season, the agency reported just over 1,000 cases so far. Increased use of flu vaccine, which is readily available to just about everyone, may have also contributed to the decline.

"Fewer cases of the respiratory syncytial virus are also being reported. The seasonal virus can be deadly for infants and young children, so less of it is an unexpected silver lining of COVID-19. Experts warn against complacency, though."

Fewer cases of the respiratory syncytial virus are also being reported. The seasonal virus can be deadly for infants and young children, so less of it is an unexpected silver lining of COVID-19. Experts warn against complacency, though.

"While we're not seeing as many severe cases of RSV right now, we are still seeing some, and parents must remain vigilant," said Suzanne Staebler, DNP. "RSV is still circulating, and it's still dangerous."

Staying alert to symptoms is good advice - as the dip is likely temporary. A rebound of new viral infections is expected when current precautionary measures and ongoing travel restrictions subside. And next season is already anticipated to be more severe than usual due to lack of exposure this year.

"The world remains laser-focused on the distribution of COVID-19 vaccinations, and rightly so. The mass vaccination effort is critical to the world's return to "normal." Yet when coronavirus fades, policymakers would do well to shift their focus toward ensuring better access to the RSV preventive treatment."

Like the flu and COVID-19, there is a preventive treatment for RSV. Getting babies access to it, however, has historically been difficult. Upward of $\underline{\mathbf{4 0} \%}$ of prescriptions for the treatment are denied by health plans for some of the most at-risk infants.

The world remains laser-focused on the distribution of COVID-19 vaccinations, and rightly so. The mass vaccination effort is critical to the world's return to "normal." Yet when coronavirus fades, policymakers would do well to shift their focus toward ensuring better access to the RSV preventive treatment. It, too, has the potential to save thousands of precious young lives.
References:

1. https://www.cdc.gov/f/u/week/y/week/yarchives2020-2021/week52.htm

2. https://instituteforpatientaccess.org/wp-content/uploads/2020/04/IfPA-ReportCard-RSV-2020-National.pdf

Disclosure: The author has no relevant disclosures.

NT

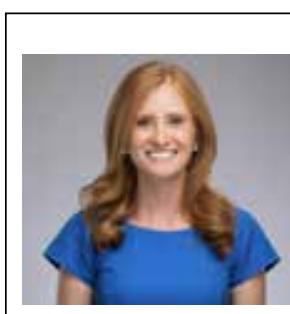

Susan Hepworth

Director

National Coalition for Infant Health

1275 Pennsylvania Ave. NW, Suite 1100A

Washington, DC 20004

Email: info@infanthealth.org

\section{National Coalition for Infant Health Values (SANE)}

Safety. Premature infants are born vulnerable. Products, treatments and related public policies should prioritize these fragile infants' safety.

Access. Budget-driven health care policies should not preclude premature infants' access to preventative or necessary therapies.

Nutrition. Proper nutrition and full access to health care keep premature infants healthy after discharge from the NICU.

Equality. Prematurity and related vulnerabilities disproportionately impact minority and economically disadvantaged families. Restrictions on care and treatment should not worsen inherent disparities. 